\title{
Combined exosomal RNA and circulating tumor DNA for epidermal growth factor mutation detection in non-small cell lung cancer
}

\author{
Chiara Lazzari $^{1}$, Vanesa Gregorc ${ }^{1}$, Maria Giulia Cangi ${ }^{2}$, Elisa Giovannetti ${ }^{3,4,5}$, Alessandra Bulotta ${ }^{1}$, \\ Mariacarmela Santarpia ${ }^{6}$
}

${ }^{1}$ Department of Oncology, Division of Experimental Medicine, IRCCS San Raffaele, Milan, Italy; ${ }^{2}$ Unit of Pathology, IRCCS San Raffaele Scientific Institute, Milan, Italy; ${ }^{3}$ Department of Medical Oncology, VU University Medical Center, Amsterdam, The Netherlands; ${ }^{4} \mathrm{CNR}-\mathrm{Nano}$, Institute of Nanoscience and Nanotechnology, Pisa, Italy; ${ }^{5}$ Cancer Pharmacology Lab, AIRC Start-Up Unit, University of Pisa, Pisa, Italy; ${ }^{6}$ Medical Oncology Unit, Department of Human Pathology of Adult and Evolutive Age "G. Barresi”, University of Messina, Messina, Italy

Correspondence to: Mariacarmela Santarpia, MD, PhD. Medical Oncology Unit, Department of Human Pathology of Adult and Evolutive Age “G. Barresi”, University of Messina, Messina, Italy. Email: msantarpia@unime.it.

Provenance: This is an invited Editorial commissioned by Section Editor Chunlin Ou (Cancer Research Institute of Central South University, Changsha, China).

Comment on: Krug AK, Enderle D, Karlovich C, et al. Improved EGFR mutation detection using combined exosomal RNA and circulating tumor DNA in NSCLC patient plasma. Ann Oncol 2018;29:700-6.

Submitted Sep 05, 2018. Accepted for publication Oct 02, 2018.

doi: $10.21037 /$ jtd.2018.10.17

View this article at: http://dx.doi.org/10.21037/jtd.2018.10.17

\section{Introduction}

The use of molecularly targeted therapies has markedly improved the survival of patients with non-small cell lung cancer (NSCLC) harboring specific oncogenic alterations, such as epidermal growth factor (EGFR) mutations, anaplastic lymphoma kinase (ALK) and ROS1 rearrangements. Hence, molecular profiling of tumors has been rapidly incorporated into clinical practice in order to define the optimal therapeutic strategy (1). Despite the initial efficacy of EGFR and ALK tyrosine kinase inhibitors (TKIs), acquired resistance to these agents inevitably occurs and patients develop progressive disease. Multiple mechanisms of acquired resistance to EGFR TKIs have been described (2), including the emergence of secondary mutations within the EGFR kinase domain, the activation of alternative signaling pathways, small-cell lung cancer transformation and epithelial to mesenchymal transition. A secondary mutation at exon 20 within the kinase domain of EGFR, the T790M gatekeeper mutation, is the most common mechanism of acquired resistance, occurring in approximately $50-60 \%$ of EGFR-TKI-resistant tumors. Osimertinib, a third-generation TKI, is the first drug to be approved for treatment of EGFR T790M mutationpositive NSCLC patients progressing after EGFR TKI therapy, thus supporting the need of tumor genotyping at the time of disease progression for evaluation of T790M and identification of eligible patients $(3,4)$.

Despite the importance of tracking dynamic evolution of tumor mutational profile over the course of treatment, tissue rebiopsy has several disadvantages in routine clinical practice, due to the accessibility of metastatic sites and the risks of patients' complications. In approximately $23 \%$ of cases, tumor tissue is not adequate to perform molecular analyses (5). Moreover, the tissue collected is obtained as a single snapshot and may not be representative of the whole genomics profile because of intratumor heterogeneity, thereby missing potential targetable gene mutations.

In recent years, liquid biopsy has emerged as a viable alternative to tissue genotyping for the assessment of tumorspecific molecular alterations in lung cancer patients (6). Indeed, blood is a dynamic mixture of tumor-derived components, including circulating cell-free tumor DNA (ctDNA), cell-free tumor RNA (ctRNA), exosomes, tumor-educated platelets (TEP), and circulating tumor cells (CTCs). Higher levels of cell-free DNA (cfDNA) 
were observed in blood from cancer patients compared to healthy individuals (7), and in those with metastatic disease compared to non-metastatic patients (7). CtDNAs originating from cancer cells, mainly through apoptosis or necrosis, have genomic profiles closely matching those of corresponding tumors, thus they can be assessed for a variety of genomic alterations, including mutations in oncogenes or tumor suppressor genes, gene amplifications or epigenetic changes. Liquid biopsy has already been integrated into clinical practice in lung cancer, where ctDNA genotyping is currently used to analyze EGFR sensitizing and T790M mutations as surrogate of tumor tissue profiling to select patients for EGFR TKI therapy. For this purpose, blood-based assays, mainly represented by real-time polymerase chain reaction (PCR)-based techniques, such as the Roche cobas ${ }^{\circledR}$ EGFR mutation test v2 for T790M, have been approved for plasma genotyping (8). However, despite the use of highly sensitive technologies, the isolation and enrichment of ctDNA remains a big challenge, due to the high degree of DNA fragmentation and its lower concentration in the circulation relative to wild-type DNA.

\section{Combined exosomal RNA (exoRNA) and circulating tumor DNA}

In order to improve detection of druggable oncogenic drivers, such as EGFR mutations, the molecular profiles gathered from ctDNA can be complemented with those from other tumor-derived materials, including CTCs or RNA derived from exosomes. In their work, Krug and collaborators evaluated the benefit of concurrent isolation of plasma exoRNA and cfDNA (exoNA) by EXO1000, a next generation sequencing (NGS) assay, for the detection of EGFR mutations compared to ctDNA analysis alone (9). In this study, matched tumor and plasma samples were collected from 84 NSCLC patients with EGFR mutations enrolled in the TIGER-X trial, a phase $1 / 2$ study of the third-generation EGFR TKI, rociletinib. ExoNA was analyzed from plasma samples and results were compared with matching tissue data and existing data from ctDNA assessed by a sensitive technology based on digital PCR, the Beads, Emulsion, Amplification, and Magnetics (BEAMing).

In order to evaluate the advantage of this technique, the study population was enriched with some cases particularly challenging for EGFR mutation detection: 50 (subgroup B) with low copy T790M ( $<10$ copies/mL), and within these, 21 (subgroup C) with intrathoracic disease only. Results showed that EGFR activating mutations and T790M were detected by exoNA with greater sensitivity $(98 \%$ and $90 \%$, respectively) compared to ctDNA ( $82 \%$ and $84 \%$, respectively). This difference significantly increased in subgroup B, where the sensitivity for EGFR activating mutations was $81 \%$ with exoNA and $58 \%$ with ctDNA $(\mathrm{P}=0.003)$, while for $\mathrm{T} 790 \mathrm{M}$ it was $61 \%$ with exoNA over $53 \%$ with ctDNA $(\mathrm{P}=0.5)$. The greatest difference was observed in subgroup $C$, where EGFR activating mutations and T790M were detected by exoNA with a sensitivity of $74 \%$ and $31 \%$, respectively, compared to $26 \%$ and $19 \%$, respectively of ctDNA ( $\mathrm{P}=0.003$ for activating mutations and $\mathrm{P}=0.5$ for $\mathrm{T} 790 \mathrm{M})$. These data strongly suggest that this platform could be particularly useful to improve EGFR mutation detection compared to the use of BEAMing alone. ExoNA could be particularly useful in those subsets of patients for which plasma tests, based exclusively on ctDNA, have demonstrated an overall low sensitivity for mutation detection, such as those with intrathoracic disease (9). The lower sensitivity for T790M detection compared to driver mutations, observed with both methods, might be related to a lower fraction of T790M alleles shed in plasma, as a consequence of intratumoral and intertumoral heterogeneity of mechanisms of resistance or due to the low tumor burden. Heterogeneity can be another responsible of negative results of EGFR mutations, especially T790M, in tissue. On the other hand, cell-free DNA fragments from multiple lesions in the same individual mix together in the peripheral blood, and therefore plasma-assays are likely to offer a wider representation of the entire genomic landscape, including primary tumor and multiple metastatic sites, compared with tissue from single biopsy. Remarkably, in the study by Krug and collaborators a significantly higher number of EGFR activating- and T790M-mutation copies were isolated using exoNA compared to ctDNA (9).

\section{Other methods to detect EGFR mutations in blood}

Based on these findings, the sensitivity for detection of EGFR mutations using exoNA appears greater compared to several highly sensitive methods described in literature. According to previous data, the sensitivity for detection of EGFR activating mutations ranged between $73 \%$ to $84 \%$ for the cobas test, $82 \%$ to $84 \%$ for the BEAMing assay, and $74 \%$ to $82 \%$ for the digital droplet PCR (ddPCR) platform $(10,11)$, while the sensitivity for T790M detection 
Table 1 Sensitivity for detection of EGFR mutations with different methods

\begin{tabular}{lcccc}
\hline Method (ref) & COBAS (10) & BEAMing (11) & ddPCR (11) & exoNA (9) \\
\hline EGFR activating mutations (\%) & $73-84$ & $82-84$ & $74-82$ & 98 \\
EGFR T790M (\%) & $64-73$ & $73-81$ & 77 & 90 \\
\hline
\end{tabular}

EGFR, epidermal growth factor; BEAMing, Beads, Emulsion, Amplification, and Magnetics; ddPCR, digital droplet polymerase chain reaction; exoNA, exosomal RNA and circulating free DNA.

in patients progressing to first-line EGFR TKIs ranged between $64 \%$ and $73 \%$ for the cobas test, $73 \%$ to $81 \%$ for BEAMing, and $77 \%$ for ddPCR platform (Table 1). However, the increase in sensitivity, using exoNA, was not associated with an increased in specificity, which is one of the main strengths of PCR-based assays. In five studies, including patients with lung cancer, where tissue genotype was considered as the reference standard, specificity for driver mutations was $96 \%$, and sensitivity $66 \%$ (11-15). Conversely, data from literature indicate that specificity for EGFR T790M is more variable (ranging from $40 \%$ to $78 \%$ ), probably due to the high rate of false negative results in tissue. These false negative results in tissue are most likely due to a selection bias from tissue biopsy as consequence of intratumor genetic heterogeneity (11).

\section{Liquid biopsy to monitor treatment}

Another objective of the study by Krug and collaborators was to assess the potential role of liquid biopsy to predict patients' response to treatment through examination of longitudinal changes of plasma EGFR mutations levels within the first two weeks of treatment. ExoNA was able to accurately identify responders and non-responders to rociletinib. The predictive value of EGFR sensitizing mutations and of T790M mutations assessed in liquid biopsy has been widely demonstrated. For example, in an exploratory analysis of the phase I AURA trial, where BEAMing digital PCR was applied to identify EGFR T790M in plasma samples, median progression free survival (PFS) and objective response rate (ORR) under osimertinib were comparable between patients with T790M-plasma positive and those with T790M-tumor positive (11), suggesting that plasma T790M is a valid biomarker for treatment decisions. However, longer median PFS (16.5 months) was observed in patients with T790M-tumor positive and plasma negative, compared to those with T790M-tumor positive and plasma positive (9.3 months) or those with T790M-tumor negative and plasma positive
(4.2 months), suggesting that plasma and tumor genotyping have complementary roles. Indeed, plasma negative is a mixture of true and false negative results, while tumor negative might be dependent by tumor heterogeneity, that it is not reflected by a single tumor biopsy. In these cases, T790M clones represent a minority of the entire tumor, thus explaining the shorter PFS and lower ORR observed in patients with T790M-plasma positive and tumor negative. Due to the risk of false negative results, if T790M is not found in plasma analysis, a new tissue biopsy is highly recommended to correctly classify patients for treatment with osimertinib. An emerging issue is the potential use of liquid biopsy as a tool to monitor treatment failure during treatment with EGFR-TKIs. Indeed, EGFR T790M mutations have been identified by ddPCR at a median time of approximately two months before RECIST progression (16). Currently, the biologic and clinical value of this finding is still unknown. The phase II APPLE trial (EORTC 1613) has been designed to compare a sequence strategy (gefitinib followed by osimertinib) with osimertinib up-front, and determine the role of liquid biopsy as a tool to define the timing of switching from first- or secondgeneration EGFR-TKIs to osimertinib. One hundred fiftysix EGFR mutated NSCLC patients will be randomized between osimertinib, or gefitinib followed by osimertinib at the time of the emergence of T790M in blood independent of RECIST progression, or gefitinib followed by osimertinib at the time of disease progression. The primary end point is PFS rate at 18 months. Recently, quantification of the T790M alleles fraction (AF) in patients receiving osimertinib has demonstrated to provide information about the dynamic evolution of tumor genome profiles and patients' outcomes (17). Three patterns of T790M changes were observed: in some patients T790M remained detectable, and was associated with progressive disease or a short-lasting stable disease, in other cases T790M became undetectable and patients developed partial response or stable disease, while in approximately $15 \%$ of samples T790M disappeared despite progression. In these patients, 
T790M probably was a minor clone, and additional acquired resistance mechanisms coexisted. Quantification of the T790M AF could represent a strategy to provide a picture of tumor heterogeneity and a predictive molecular biomarker to be exploited for treatment decisions.

\section{Comparison of exoRNA and circulating tumor DNA}

Exosomes, which are actively released by living tumor cells, contain intact and good quality nucleic acids for molecular analysis and other tumor-derived materials, including RNA, DNA and proteins, thereby offering a more comprehensive assessment of cancer biomarkers. Moreover, exosomes are tumor specific and are protected from degradation by a lipid bilayer membrane, in contrast to ctDNA, that is released by necrotic/apoptotic cells. Therefore, mRNA isolated from exosomes might be present at higher concentrations into circulation, and analysis of exosomal mRNA might be advantageous, especially in patients with limited amounts of detectable ctDNA. In this context, the combined detection of cfDNA and exosomal nucleic acids analyze two different biological processes of the tumor and could further enhance T790M mutation detection. There are several limitations in the use of exosomes as biomarkers in the clinical setting. First, diverse methods are used for exosome isolation/purification and the different techniques could slightly affect exosomal contents. Second, platelet-derived exosomes constitute the greatest percentage of extracellular vesicles in blood and levels of platelets vary depending on age, stress, and infection status, as well as many other diseases (18). These conditions might affect the content of exosomes and the sensitivity of different assays. Finally, discrepancies in the findings from some important biomarker studies on exosomes could also be attributable to differences between patient cohorts, sample sizes, or reagent specificity, as recently reviewed by $\mathrm{Xu}$ and collaborators (19). However, given the urgent need for biomarkers in the clinical setting, these studies warrant further research using uniform methodologies and proper technical validation in larger sample cohort.

In conclusions, combining exoRNA and ctDNA increased the sensitivity for EGFR mutations detection, especially in those cases with intrathoracic disease only and low levels of nucleic acids, and it could be considered as an alternative option preferable to ctDNA. This sensitive method could also offer the potential for investigating novel biomarkers correlated to response or resistance to oncogene-directed therapies.

\section{Acknowledgements}

None.

\section{Footnote}

Conflicts of Interest: The authors have no conflicts of interest to declare.

\section{References}

1. Rosell R, Karachaliou N. Large-scale screening for somatic mutations in lung cancer. Lancet 2016;387:1354-6.

2. Sequist LV, Waltman BA, Dias-Santagata D, et al. Genotypic and histological evolution of lung cancers acquiring resistance to EGFR inhibitors. Sci Transl Med 2011;3:75ra26.

3. Mok TS, Wu YL, Ahn MJ, et al. Osimertinib or PlatinumPemetrexed in EGFR T790M-Positive Lung Cancer. N Engl J Med 2017;376:629-40.

4. Santarpia M, Liguori A, Karachaliou N, et al. Osimertinib in the treatment of non-small-cell lung cancer: design, development and place in therapy. Lung Cancer (Auckl) 2017;8:109-25.

5. Sundaresan TK, Sequist LV, Heymach JV, et al. Detection of T790M, the Acquired Resistance EGFR Mutation, by Tumor Biopsy versus Noninvasive Blood-Based Analyses. Clin Cancer Res 2016;22:1103-10.

6. Santarpia M, Liguori A, D'Aveni A, et al. Liquid biopsy for lung cancer early detection. J Thorac Dis 2018;10:S882-97.

7. Leon SA, Shapiro B, Sklaroff DM, et al. Free DNA in the serum of cancer patients and the effect of therapy. Cancer Res 1977;37:646-50.

8. cobas EGFR Mutation Test v2. Available online: https://www.fda.gov/Drugs/InformationOnDrugs/ ApprovedDrugs/ucm504540.htm

9. Krug AK, Enderle D, Karlovich C, et al. Improved EGFR mutation detection using combined exosomal RNA and circulating tumor DNA in NSCLC patient plasma. Ann Oncol 2018;29:2143.

10. Thress KS, Brant R, Carr TH, et al. EGFR mutation detection in ctDNA from NSCLC patient plasma: A crossplatform comparison of leading technologies to support the clinical development of AZD9291. Lung Cancer 2015;90:509-15.

11. Oxnard GR, Thress KS, Alden RS, et al. Association Between Plasma Genotyping and Outcomes of Treatment With Osimertinib (AZD9291) in Advanced Non-Small- 
Cell Lung Cancer. J Clin Oncol 2016;34:3375-82.

12. Kukita Y, Uchida J, Oba S, et al. Quantitative identification of mutant alleles derived from lung cancer in plasma cellfree DNA via anomaly detection using deep sequencing data. PLoS One 2013;8:e81468.

13. Douillard JY, Ostoros G, Cobo M, et al. Gefitinib treatment in EGFR mutated caucasian NSCLC: circulating-free tumor DNA as a surrogate for determination of EGFR status. J Thorac Oncol 2014;9:1345-53.

14. Li X, Ren R, Ren S, et al. Peripheral blood for epidermal growth factor receptor mutation detection in non-small cell lung cancer patients. Transl Oncol 2014;7:341-8.

15. Mok T, Wu YL, Lee JS, et al. Detection and Dynamic Changes of EGFR Mutations from Circulating Tumor DNA as a Predictor of Survival Outcomes in NSCLC Patients Treated with First-line Intercalated Erlotinib and

Cite this article as: Lazzari C, Gregorc V, Cangi MG, Giovannetti E, Bulotta A, Santarpia M. Combined exosomal RNA and circulating tumor DNA for epidermal growth factor mutation detection in non-small cell lung cancer. J Thorac Dis 2018;10(Suppl 33):S4023-S4027. doi: 10.21037/jtd.2018.10.17
Chemotherapy. Clin Cancer Res 2015;21:3196-203.

16. Zheng D, Ye X, Zhang MZ, et al. Plasma EGFR T790M $\mathrm{ctDNA}$ status is associated with clinical outcome in advanced NSCLC patients with acquired EGFR-TKI resistance. Sci Rep 2016;6:20913.

17. Moran T, Felip E, Bosch-Barrera J, et al. Monitoring EGFR-T790M mutation in serum/plasma for prediction of response to third-generation EGFR inhibitors in patients with lung cancer. Oncotarget 2018;9:27074-86.

18. Best MG, Sol N, In't Veld S, et al. Swarm IntelligenceEnhanced Detection of Non-Small-Cell Lung Cancer Using Tumor-Educated Platelets. Cancer Cell 2017;32:238-52.e9.

19. Xu R, Rai A, Chen M, et al. Extracellular vesicles in cancer - implications for future improvements in cancer care. Nat Rev Clin Oncol 2018;15:617-38. 